\title{
A NOTE ON THE CONTINUITY OF PROJECTION MATRICES WITH APPLICATION TO THE ASYMPTOTIC DISTRIBUTION OF QUADRATIC FORMS
}

Abstract. This paper investigates the continuity of projection matrices and illustrates an important application of this property to the derivation of the asymptotic distribution of quadratic forms. We give a new proof and an extension of a result of Stewart (1977).

An important result in statistics concerning the distribution of quadratic forms is the following: if $X$ is a $k \times 1$ vector having a multivariate normal distribution with mean vector $\mu$ and identity covariance matrix, and if $P_{B}$ is an idempotent matrix of rank $p$ then $X^{\prime} P_{B} X$ is $\chi^{2}(p, \delta)$, where $\delta=\mu^{\prime} P_{B} \mu$ (see for instance Muirhead (1982), Theorem 1.4.5).

Sometimes we are interested in an asymptotic version of this result (examples are given below): (i) the $k \times 1$ random vector $X_{T}$ indexed by $T$ converges in distribution to a multivariate normal random variable with unknown mean $\mu$ and identity covariance matrix $I_{k}$; (ii) the $k \times n(n \leq k)$ matrix $\widehat{B}_{T}$ converges in probability to the $k \times n$ matrix $B$ (and we write $\left.\operatorname{plim}_{T \rightarrow \infty} \widehat{B}_{T}=B\right)$. In this application $\widehat{B}_{T}$ has rank $n$ with probability 1 for all but a finite number of $T$. Let $P_{\widehat{B}_{T}}=\widehat{B}_{T}\left(\widehat{B}_{T}^{\prime} \widehat{B}_{T}\right)^{-1} \widehat{B}_{T}^{\prime}$. We want to find the asymptotic distribution of $X_{T}^{\prime} P_{\widehat{B}_{T}} X_{T}$ as $T \rightarrow \infty$. If the mapping $\widehat{B}_{T} \rightarrow P_{\widehat{B}_{T}}$ is continuous we can conclude that $X_{T}^{\prime} P_{\widehat{B}_{T}} X_{T}$ has an asymptotically noncentral chi-square distribution with $n$ degrees of freedom and noncentrality parameter $\mu^{\prime} P_{B} \mu$ (Muirhead (1982), Theorem 1.4.5). However, if $n>p=\operatorname{rank}(B)$ for all but a finite number of $T$, then $X_{T}^{\prime} P_{\widehat{B}_{T}} X_{T}$ often has an asymptotically noncentral chi-square distribution with (again) $n$ degrees of freedom (rather than $p$ ) and noncentrality parameter $\mu^{\prime}\left(\operatorname{plim}_{T \rightarrow \infty} P_{\widehat{B}_{T}}\right) \mu$. Two examples where such situation arises are given below.

2000 Mathematics Subject Classification: 54C05, 15A03, 62E20.

Key words and phrases: projection matrix, distribution of quadratic forms. 
ExAmple 1. Consider a single linear structural equation

$$
y_{1}=Y_{2} \beta+Z_{1} \gamma+u,
$$

where $y_{1}$ and $Y_{2}$ are a $T \times 1$ vector and a $T \times n$ matrix of endogenous variables, $Z_{1}$ is a $T \times k_{1}$ matrix of exogenous variables, and $\beta$ and $\gamma$ are $n \times 1$ and $k_{1} \times 1$ vectors of parameters. The reduced form equation for $Y_{2}$ is

$$
Y_{2}=Z_{1} \Phi_{2}+Z_{2} \Pi_{2}+V_{2}
$$

where $Z_{2}$ is a $T \times k_{2}$ matrix of exogenous variables not included in the structural equation, and $\Phi_{2}$ and $\Pi_{2}$ are matrices of parameters of dimension $k_{1} \times n$ and $k_{2} \times n$ respectively. We assume throughout that $k_{2}>n$. Inserting the reduced form (2) into the structural equation (1) gives

$$
y_{1}=Z_{1} \phi_{1}+Z_{2} \pi_{1}+v_{1},
$$

where the parameter $\left(\phi_{1}, \pi_{1}\right)$ and the error term $v_{1}$ satisfy the compatibility conditions:

$$
\begin{aligned}
& \pi_{1}=\Pi_{2} \beta, \\
& \phi_{1}=\gamma+\Phi_{2} \beta, \\
& v_{1}=u+V_{2} \beta .
\end{aligned}
$$

Together, equations (2) and (3) form a multivariate linear model (MLM)

$$
\left[y_{1}, Y_{2}\right]=Z_{1}\left[\phi_{1}, \Phi_{2}\right]+Z_{2}\left[\pi_{1}, \Pi_{2}\right]+\left[v_{1}, V_{2}\right]
$$

with restrictions on its coefficients and its error components. Assume that the following limits hold jointly:

(a) $\widehat{Q}_{T}=T^{-1} Z_{2}^{\prime} M_{Z_{1}} Z_{2}$ and $\operatorname{plim}_{T \rightarrow \infty} \widehat{Q}_{T}=Q$, where $Q$ is a fixed, finite, positive definite $k_{2} \times k_{2}$ matrix, and $M_{Z_{1}}=I_{T}-Z_{1}\left(Z_{1}^{\prime} Z_{1}\right)^{-1} Z_{1}^{\prime}$;

(b) $\widehat{\Omega}_{T}=T^{-1} S$ and $\operatorname{plim}_{T \rightarrow \infty} \widehat{\Omega}_{T}=\Omega$, where $S=Y^{\prime} M_{Z} Y, Y=\left[y_{1}, Y_{2}\right]$, $Z=\left[Z_{1}, Z_{2}\right]$ and $M_{Z}=I_{T}-Z\left(Z^{\prime} Z\right)^{-1} Z^{\prime}$

(c) $T^{\frac{1}{2}}(\widehat{\Pi}-\Pi) \stackrel{L}{\rightarrow} N\left(0, Q^{-1} \otimes \Omega\right)$, where $\Pi=\left[\pi_{1}, \Pi_{2}\right]$ and $\widehat{\Pi}$ is the OLS estimator of $\Pi$ in $(7)$;

and suppose one is interested in the vector of coefficients $\beta$, and wants to test whether equation (4) is satisfied. In a generalized method of moments framework, one can base such a test on the statistic

$$
\mathcal{T}=\widetilde{\pi}_{1}^{\prime}\left(I_{k_{2}}-P_{\widetilde{\Pi}_{2}}\right) \widetilde{\pi}_{1}=\widehat{u}^{\prime} Z\left(Z^{\prime} Z\right)^{-1} Z^{\prime} \widehat{u},
$$

where $\left[\widetilde{\pi}_{1}, \widetilde{\Pi}_{2}\right]=\widehat{Q}_{T}^{1 / 2}\left[\widehat{\pi}_{1}, \widehat{\Pi}_{2}\right]$ and $\left[\widehat{\pi}_{1}, \widehat{\Pi}_{2}\right]$ is the ordinary least squares estimator of $\left[\pi_{1}, \Pi_{2}\right]$ in (7). Forchini (2003) shows that if (4) holds then

$$
\frac{T \mathcal{T}}{\omega_{11.2}\left(1+\left(\beta_{1}^{*}\right)^{\prime} \beta_{1}^{*}\right)} \stackrel{L}{\rightarrow} \chi^{2}\left(k_{2}-n\right)
$$


independently of the rank of $\Pi_{2}$. In the expression above we have used

$$
\begin{aligned}
\Omega & =\left(\begin{array}{ll}
\omega_{11} & \omega_{21}^{\prime} \\
\omega_{21} & \Omega_{22}
\end{array}\right), \\
\omega_{11.2} & =\omega_{11}-\omega_{12} \Omega_{22}^{-1} \omega_{21}, \\
\beta^{*} & =\left(\Omega_{22}^{1 / 2} \beta-\Omega_{22}^{-1 / 2} \omega_{21}\right) / \omega_{11.2}^{1 / 2},
\end{aligned}
$$

and $\beta^{*}=\left(\beta_{1}^{* \prime}, \beta_{2}^{* \prime}\right)^{\prime}$ is partitioned conformably to

$$
\pi_{1}=\left(\Pi_{21}, \Pi_{22}\right)\left(\begin{array}{c}
\beta_{1} \\
\beta_{2}
\end{array}\right)
$$

where $\operatorname{rank}\left(\Pi_{2}\right)=\operatorname{rank}\left(\Pi_{21}\right)=r \leq n$.

EXAMPLE 2. A similar situation occurs with asymptotically uncooperative regressors (Schmidt (1976), pp. 85-88): consider the case

$$
Y_{T} \sim W_{T} \beta+U_{T}, \quad U_{T} \sim N\left(0, \sigma^{2} I_{T}\right),
$$

where the $i$ th row of $W_{T}$ is $\left(1, \lambda^{i}\right)$ with $|\lambda|<1$ and $\sigma^{2}$ known. When testing whether $\beta=0$ one considers the statistic

$$
\sigma^{-2} U_{T}^{\prime} P_{W_{T}} U_{T}=X_{T}^{\prime} X_{T},
$$

where $X_{T}=\sigma^{-1}\left(W_{T}^{\prime} W_{T}\right)^{-1 / 2} W_{T}^{\prime} U_{T} \sim N\left(0, \sigma^{2} I_{2}\right)$. This quantity has a chisquare distribution with $2\left(=\operatorname{rank}\left(P_{X_{T}}\right)\right)$ degrees of freedom. Note however that $T^{-1} W_{T}^{\prime} W_{T} \rightarrow\left(\begin{array}{ll}1 & 0 \\ 0 & 0\end{array}\right)$ so that $\operatorname{rank}\left(P_{\lim _{T \rightarrow \infty} X_{T}}\right)=1$.

These results are surprising: the limiting distribution of $X_{T}^{\prime} P_{\widehat{B}_{T}} X_{T}$ does not depend on $p$. To understand why, we need to look at the properties of the mapping $\widehat{B}_{T} \mapsto P_{\widehat{B}_{T}}$. Let $B_{T}$ be a sequence of $k \times n(n \leq k)$ matrices converging to the $k \times n$ matrix $B$, and let $P_{B_{T}}=B_{T} B_{T}^{\dagger}$ and $P_{B}=B B^{\dagger}$ be the projections on the spaces spanned by the columns of $B_{T}$ and $B$ respectively. The matrices $B_{T}^{\dagger}$ and $B^{\dagger}$ are the Moore-Penrose generalised inverses of $B_{T}$ and $B$.

Corollary 3.5 of Stewart (1977) shows that a necessary and sufficient condition for $\lim _{T \rightarrow \infty} B_{T}^{\dagger}=B^{\dagger}$ is that $\operatorname{rank}\left(B_{T}\right)=\operatorname{rank}(B)$ for all but a finite number of $T$. Given the uniqueness of the Moore-Penrose generalised inverse, it follows that a necessary and sufficient condition for $\lim _{T \rightarrow \infty} P_{B_{T}}=$ $P_{B}$ is that $\operatorname{rank}\left(B_{T}\right)=\operatorname{rank}(B)$ for all but a finite number of $T$. In this note we give a new direct proof of this result, and give an indication of what happens when $\operatorname{rank}\left(B_{T}\right)>\operatorname{rank}(B)$ for all but a finite number of $T$.

Precisely, we have:

Proposition 1. Let $B_{T}$ be a sequence of matrices converging to the matrix $B$. 
(i) $\lim _{T \rightarrow \infty} P_{B_{T}}=P_{B}$ if and only if $\operatorname{rank}\left(B_{T}\right)=\operatorname{rank}(B)$ for all but a finite number of $T$.

(ii) If $\operatorname{rank}\left(B_{T}\right)>\operatorname{rank}(B)$ for all but a finite number of $T$, then the space spanned by the columns of $B$ is contained in the space spanned by $\lim _{T \rightarrow \infty} P_{B_{T}}$.

Thus, the space spanned by the columns of $\operatorname{plim}_{T \rightarrow \infty} P_{\widehat{B}_{T}}$ is larger than the space spanned by the columns of $P_{B}=P_{\text {plim }} \rightarrow \infty \widehat{B}_{T}$. The degrees of freedom of the resulting chi-square distribution are not affected by the rank of $B$ because the space spanned by the columns of $P_{\widehat{B}_{T}}$ does not collapse to that spanned by the columns of $B$.

Proof of Proposition 1. Consider a sequence $B_{T} \rightarrow B$. Without loss of generality we can assume that $\operatorname{rank}\left(B_{T}\right)=g \geq p=\operatorname{rank}(B)$. Then write

$$
B_{T} B_{T}^{\prime}=H_{T}\left(\begin{array}{cc}
\Lambda_{T} & 0 \\
0 & 0
\end{array}\right) H_{T}^{\prime}
$$

where $H_{T}$ is a $k \times k$ orthogonal matrix and $\Lambda_{T}$ is a $g \times g$ matrix which contains the $g$ nonzero eigenvalues of $B_{T} B_{T}^{\prime}$ in descending order. Partition $H_{T}$ as $H_{T}=\left(H_{1 T}, H_{2 T}\right)$, where $H_{1 T}$ is $k \times g$ and $H_{2 T}$ is $k \times(k-g)$. The space spanned by the columns of $B_{T}$ is spanned by the eigenvectors corresponding to $B_{T} B_{T}^{\prime}$, i.e. the columns of $H_{1 T}$, so we can write

$$
P_{B_{T}}=H_{1 T} H_{1 T}^{\prime} \text {. }
$$

Now, $P_{B_{T}} \rightarrow P_{B}$ if and only if every subsequence of $P_{B_{T}}$ has a subsequence which converges to $P_{B}$.

Now consider an arbitrary subsequence $P_{B_{T_{q}}}$. The elements of $H_{T_{q}}$ and $\Lambda_{T_{q}}$ are bounded above uniformly in $T$, so there is a subsequence such that $H_{T_{q_{s}}}$ and $\Lambda_{T_{q_{s}}}$ converge to some matrices $H=\left(H_{1}, H_{2}\right)$ and $\Lambda$, and $B=H\left(\begin{array}{ll}\Lambda & 0 \\ 0 & 0\end{array}\right) H^{\prime}$, where $H$ is orthogonal and $\Lambda$ is diagonal with $g$ nonzero diagonal elements.

Consider the case where $g=p$. Then $H_{T_{q_{s}}} \rightarrow H$ and $H_{1 T_{q_{s}}} \rightarrow H_{1}$, $\Lambda_{T_{q_{s}}} \rightarrow \Lambda(p \times p)$ so that as $q \rightarrow \infty$,

$$
P_{B_{T_{q_{s}}}}=H_{1 T_{q_{s}}} H_{1 T_{q_{s}}}^{\prime} \rightarrow H_{1} H_{1}^{\prime}=P_{B} .
$$

If $g>p$, it is still true that $H_{T_{q_{s}}} \rightarrow H$ and $H_{1 T_{q_{s}}} \rightarrow H_{1}$. Moreover $\Lambda_{1 T_{q_{s}}} \rightarrow$ $\Lambda=\left(\begin{array}{cc}\Lambda_{*} & 0 \\ 0 & 0\end{array}\right)$ so that

$$
P_{B_{T_{q_{s}}}}=H_{1 T_{q_{s}}} H_{1 T_{q_{s}}}^{\prime} \rightarrow H_{1} H_{1}^{\prime}
$$

But $P_{B}$ is the projection on the space spanned by the eigenvector associated to the nonzero eigenvalues of $B B^{\prime}$, so partitioning $H_{1}=\left(H_{11}, H_{12}\right)$, where $H_{11}$ is $k \times p$ and $H_{12}$ is $k \times(k-p)$, we have

$$
P_{B}=H_{11} H_{11}^{\prime}
$$


so that

$$
\lim _{T \rightarrow \infty}\left(P_{B_{T}}-P_{B}\right)=H_{12} H_{12}^{\prime} \neq 0 .
$$

Note that $H_{12} H_{12}^{\prime}$ is itself a projection into the space spanned by the columns of $B_{T}$ and orthogonal to the space spanned by the columns of $B$.

\section{References}

G. Forchini (2003), Testing the relevance of instrumental variables in structural estimation, preprint.

R. J. Muirhead (1982), Aspects of Multivariate Statistical Theory, Wiley, New York.

P. Schmidt (1976), Econometrics, Dekker, New York.

G. W. Stewart (1977), On the perturbation of pseudo-inverses, projections and linear least squares problems, SIAM Rev. 19, 634-662.

Department of Econometrics and Business Statistics

Faculty of Business and Economics

Monash University

Clayton, VIC 3800, Australia

Received on 8.9.2003;

revised version on 7.10 .2004 